\title{
Umbilical artery velocity and adverse neonatal outcome in sudanese pregnant women with severe preeclampsia
}

\author{
Moawia Bushra Gameraddin ${ }^{1}$, Amel Alla Gabo ${ }^{2}$
}

\author{
${ }^{1}$ Department of Diagnostic Radiologic Technology, Faculty of Applied Medical Sciences, Taibah University, Al- \\ Madinah Al Munawwarah, Kingdom of Saudi Arabia \\ ${ }^{2}$ Faculty of Radiological Sciences and Medical Imaging, Alzaiem Alazhari University, Sudan
}

Received: 28 September 2016

Accepted: 24 October 2016

\section{*Correspondence: \\ Dr. Moawia Bushra Gameraddin, \\ E-mail: m.bushra@yahoo.com}

Copyright: ( ) the author(s), publisher and licensee Medip Academy. This is an open-access article distributed under the terms of the Creative Commons Attribution Non-Commercial License, which permits unrestricted non-commercial use, distribution, and reproduction in any medium, provided the original work is properly cited.

\begin{abstract}
Background: Doppler umbilical artery waveform is an important imaging predictor of adverse neonatal outcome in patients with severe preeclampsia. The present study aims to evaluate the adverse neonatal outcome associated with abnormal umbilical artery velocity in patients with severe preeclampsia.

Methods: In a prospective descriptive analytical hospital-based study, umbilical artery velocity and adverse fetal outcome of (104) patients with severe preeclampsia were evaluated by Doppler ultrasound. The study was conducted through the period (February to August 2015) in obstetric Sudanese population.

Results: The umbilical artery end diastolic flow was reduced in $20.2 \%$, absent in $5.8 \%$, reversed in $1.9 \%$ and normal in $72.1 \%$ of the study population. The end diastolic velocity was significantly abnormal and associated with low birth weight $(\mathrm{P}$-value $=0.001)$, early neonatal death $(\mathrm{P}$-value $=0.00)$, fetal respiratory distress $(\mathrm{P}$-value $=0.00)$ and Apgar score $(\mathrm{P}$-value $=0.00)$. The abnormal end diastolic flow was not statistically associated with emergency caesarean delivery $(\mathrm{P}$ value $=0.275)$.

Conclusions: The end diastolic flow velocity of the umbilical artery was significantly associated with adverse neonatal and perinatal outcomes in Sudanese pregnant cases with severe preeclampsia.
\end{abstract}

Keywords: Adverse, Preeclampsia, Sudanese, Umbilical artery, Velocity

\section{INTRODUCTION}

Hypertensive disorders of pregnancy represent the most common medical complication of pregnancy and remain one of the most significant and important unsolved problems in obstetrics. They complicate 5 to 10 percent of all pregnancies, and together with hemorrhage and infection, they form a deadly triad, which contribute greatly to maternal morbidity and mortality rates. With hypertension, the pre-eclampsia syndrome is the most dangerous. The World Health Organization systematically reviews maternal mortality worldwide. There was 16 percent of maternal deaths in developed countries were due to hypertensive disorders. This percentage is larger than three other leading causes: hemorrhage $-13 \%$, abortion $-8 \%$, and sepsis $-2 \%$.
Maternal mortality from hypertensive disorders worldwide accounts for approximately 100.000 deaths per year, reaching a rate of 70-120 death per 100.000 maternities in developing countries. ${ }^{2}$

The overall perinatal mortality in preeclampsia is around $35 / 1000$ total birth, but may reach $160 / 1000$ maternities in severe disease, while the overall perinatal mortality in normotensive women is $13 / 1000$ total birth. The most important factor is the gestational age at time of delivery; the perinatal mortality may reach 40 percent if the gestational age at delivery is less than 28 weeks gestation. Iatrogenic prematurity in hypertensive disorders of pregnancy accounts for $18 \%$ of all premature babies. These small babies not only develop neonatal complications of prematurity, but also develop health 
implications in adult life including high risk of hypertension, heart diseases, diabetes, learning disabilities and low intelligence. ${ }^{3}$

Doppler ultrasound enables a better understanding of the hemodynamic changes, therefore becomes one of the most effective clinical tools for assessing fetomaternal vasculature in high risk pregnancies. It can be credited for causing a significant reduction in perinatal morbidity and mortality. ${ }^{4}$ Doppler ultrasound of umbilical artery have revealed significant diagnostic efficacy in demonstrating fetal compromise in pregnancies complicated with preeclampsia and fetal growth restriction. Umbilical artery waveform is a good indicator of fetal compromise. This is the only ante-partum fetal test that has shown level one evidence of effectiveness. However, its use should be integrated with other current fetal monitoring test and the final management should be guided by additional clinical considerations such as the gestational age, maternal and fetal status and obstetrical conditions. ${ }^{5}$

This study aimed to determine the relationship between umbilical artery end diastolic velocity (EDF) and adverse neonatal outcome in Sudanese women who developed severe preeclampsia; to detect and characterizes adverse neonatal complications associated with severe preeclampsia and to assess the value of end diastolic flow velocities (EDF) in the detection and management of severe preeclampsia. The appearance of end-diastolic flow velocities (EDV) in the umbilical artery (UA), in this study taken, has been associated with early neonatal death (END), birth weight, respiratory distress syndrome (RDS) and mode of delivery. The hypothesis in this study is that umbilical artery Doppler has a major role in assessment of risk of adverse neonatal outcome in patients with preeclampsia. That is to say that progressive reduction in the diastolic component of umbilical artery flow will mirror the risk and severity of potential fetal compromise.

\section{METHODS}

A prospective descriptive analytical hospital-based study, deals with umbilical artery end diastolic velocity (EDV) Doppler measurements and adverse neonatal outcome in severe pre-eclampsia in obstetric Sudanese population. Data has been collected from antenatal clinics in Soba University Teaching Hospital (SUTH) and Omdurman Maternity Teaching Hospital (OMTH) through the period (February to August 2015).

\section{Study population}

The participants of the study were pregnant women of 28th weeks to 41th gestational age onward. The inclusion criteria were Sudanese pregnant women with severe preeclampsia. The exclusion criteria were:

- Pregnant women with multiple pregnancies,

- Pregnant women with coexistent medical disease,
- $\quad$ Pregnant women with congenital malformation and - $\quad$ Pregnant women with umbilical artery pathology.

The sample size was 104 participants who were selected through non-probability sampling technique (Quota sampling). A data collection sheet was designed to include all study variables in form of questions.

\section{The ultrasound (US) examination}

Adequate pulsed Doppler studies of the fetal umbilical artery were performed by experienced sonologists in 104 subjects in the 3rd trimester of pregnancy. US equipments used are GE health care model No 5268207 class 1, Toshiba Nemio 20 and Siemens model No G 60S. They were pulsed Doppler equipments with multifrequency curvilinear array transducers (3.5-5 MHZ) which have variable focal zones capability, using proper gain control setting. To improve the color resolution the frame rate was adjusted and wall filter was increased to remove motion artifacts. The velocity range of display is governed by pulsed repetition frequency. Doppler US examination was performed in a semi-recumbent position with a slight left lateral tilt, this minimize the risk of developing supine hypotension syndrome.

A simple Doppler probe was placed on the abdomen of the patient and the beam was randomly directed into the uterine cavity. Preliminary real-time 2 dimensional (2-D) U/S examinational was firstly performed to identify the fetal umbilical artery structure and obtain the standard planes. Umbilical artery was identified by B. mode within the amniotic fluid by the presence of parallel line echoes which display pulsatile activity on real time image. Confirmation by color Doppler was done, and then flow velocity waveforms were recorded using pulsed Doppler. Sampling of the umbilical artery waveforms was performed at any point along the vessel, either from free loop of cord, at the placental insertion or close to the umbilical insertion. The characteristic arterial spectral waveform from the umbilical artery was obtained using continuous wave Doppler ultrasound. The angle of insonation was kept at 55 degrees in all cases. The average value of flow velocity waveform was recorded.

The researchers evaluated the velocity of the umbilical artery in four groups of pregnancy, those with normal end diastolic velocities, reduced end diastolic velocities, absent end diastolic velocities and reverse end diastolic velocities, these measurements were taken during the period $28^{\text {th }}$ and $41^{\text {th }}$ weeks of gestation.

Only the results of the last Doppler examination that performed within 7 days of delivery were considered in the correlation with neonatal outcome. The abnormal Doppler velocities was defined as end diastolic flow velocity lesser than 95th percentile for gestational age or absent or reverse end diastolic velocity waveforms of umbilical artery. 
Neonatal outcome was assessed by; mortality and morbidity in form of Apgar score of less than 7 at 5 minutes, Small for Gestational Age (SGA), admission to Special Care Baby Unit (SCBU), respiratory distress syndrome (RDS) and END.

\section{Statistical analysis}

Statistical Package for Social Sciences (SPSS), version 21 was used in conducting all data processing and statistical analysis in this study. Chi-square test was applied to estimate the relation of umbilical artery EDV with onset of pre-eclampsia, early neonatal death, birth weight, Apgar score and admission to the hospital. Percentage and descriptive statistics were used to describe the maternal characteristics. P-value $<0.5$ considered significant indicator.

\section{RESULTS}

A total of 104 patients with severe preeclampsia have been scanned with ultrasound during the study period. The maternal age was categorized in to three groups (figure 1). The characteristics of EDF of the umbilical artery were summarized in Table 1 . It was normal in $72.1 \%$ of the study population, reduced $20.2 \%$, absent $5.8 \%$ and reversed in $1.9 \%$.The maternal and pregnancy characteristics are summarized in Table 1.

The relationship between time of onset of pre-eclampsia and UA Doppler EDF velocities had been demonstrated in Table 2. Out of 75 patients with normal EDF velocities; 12 patients (16\%) had early onset preeclampsia 20-30 weeks gestation, 27 patients (36\%) with onset of pre-eclampsia between 30-36 weeks gestation and 36 patients $(48 \%)$ had late onset pre-eclampsia. Out of the 21 patients with reduced EDF velocities 7 patients (33.3\%), 9 patients $(42.9 \%)$ and 5 patients $(23.8 \%)$ had preeclampsia onset at 20-30 weeks, 30-36 weeks and 3641 weeks, respectively. Out of the 6 patients with absent EDF velocities, 3 patients $(50 \%), 2$ patients $(33.3 \%)$ and one patient $(16.7 \%)$ had preeclampsia onset at 20-30 weeks, 30-36 weeks and 36-41weeks, respectively. All patients with reversed EDF velocities 92 patients (100\%), developed preeclampsia early in gestation i.e. 20-30 week's gestation.

Table 1: Characteristics of patients and evaluation of UA Doppler EDF.

\begin{tabular}{|lll|}
\hline Characteristic & Frequency & Percent \% \\
\hline Parity & & \\
\hline Primipara & 37 & 35.6 \\
\hline Multiparous & 42 & 40.4 \\
\hline Nulliparus & 25 & 24.0 \\
\hline GA & & \\
\hline 28-32weeks & 15 & 14.4 \\
\hline 32-36weeks & 41 & 39.4 \\
\hline 37-41weeks & 48 & 46.2 \\
\hline Mode of delivery & & \\
\hline Vaginal & 39 & 37.5 \\
\hline Emergency Caesarian & 65 & 62.5 \\
\hline End diastolic flow & & \\
\hline Normal & 75 & 72.1 \\
\hline Reduced & 21 & 20.2 \\
\hline Absent & 6 & 5.8 \\
\hline Reversed & 2 & 1.9 \\
\hline
\end{tabular}

Table 2: Relationship between time of onset of pre-eclampsia and UA Doppler EDF velocities.

\begin{tabular}{|c|c|c|c|c|c|c|c|c|c|c|}
\hline \multicolumn{11}{|l|}{ EDF } \\
\hline & GA & $\begin{array}{l}\text { Normal } \\
\mathrm{n}=12\end{array}$ & & $\begin{array}{l}\text { Reduce } \\
n=7\end{array}$ & & $\begin{array}{l}\text { Absent } \\
n=3\end{array}$ & & $\begin{array}{l}\text { Rever } \\
n=2\end{array}$ & & Total \\
\hline \multirow{3}{*}{ Onset } & 20-30 weeks & $16 \%$ & 12 & $33.3 \%$ & 7 & $50 \%$ & 3 & $100 \%$ & 2 & 24 \\
\hline & $30-36$ weeks & $36 \%$ & 27 & $42.9 \%$ & 9 & $33.3 \%$ & 2 & $0 \%$ & 0 & 38 \\
\hline & $36-41$ weeks & $48 \%$ & 36 & $23.8 \%$ & 5 & $16.7 \%$ & 1 & $0 \%$ & 0 & 42 \\
\hline Total & & & 75 & & 21 & & 6 & & 2 & 104 \\
\hline
\end{tabular}

Table 3: Relationship between UA Doppler EDF velocities and mode of delivery.

\begin{tabular}{|c|c|c|c|c|c|c|c|}
\hline & \multicolumn{6}{|c|}{ Mode of delivery } & \multirow{7}{*}{ p-value } \\
\hline & Vaginal & & & Em & & Total & \\
\hline \multirow{5}{*}{ EDF } & Normal & 32 & $42.7 \%$ & 43 & $57.3 \%$ & 75 & \\
\hline & Reduced & 6 & $28.6 \%$ & 15 & $71.4 \%$ & 21 & \\
\hline & Absent & 1 & $16.7 \%$ & 5 & $83.3 \%$ & 6 & \\
\hline & Reversed & 0 & $0 \%$ & 2 & $100 \%$ & 2 & \\
\hline & Total & 39 & & 43 & & 104 & \\
\hline
\end{tabular}


The relationship between UA Doppler EDF velocimetry and mode of delivery was shown in Table 3 . There were 32 patients $(42.7 \%)$ of vaginal deliveries were in the normal EDF group. 6 patients $(28.6 \%)$ were in the reduced EDF group, while one patient $(16.7 \%)$ was in the absent EDF group. No patient delivered vaginally in the reversed EDF group. A total of 43 patients (57.3\%) of normal EDF group were delivered by emergency C/S, 15 patients $(71.4 \%)$ of reduced EDF group were delivered by emergency $\mathrm{C} / \mathrm{S}$, and 5 patients $(83.3 \%)$ of the absent EDF group were delivered by emergency $\mathrm{C} / \mathrm{S}$. All patients $(100 \%)$ of reversed EDF group were delivered by emergency caesarean section.

The relationship between UA Doppler EDF velocimetry and neonatal birth weight was shown in Table 4. Out of the 75 patients with normal EDF velocities; 58 neonates $(77.3 \%)$ had normal birth weight and 17 neonates (22.6\%) were small for gestational age (SGA). Out of the 21 patients with reduced EDF, 14 neonates $(66.7 \%)$ had normal birth weight, 5 neonates $(23.8 \%)$ were small for gestational age (SGA) while 2 neonates $(9.5 \%)$ were large for gestational age (LGA). No neonates $(0 \%)$ in patients with absent EDF had normal birth weight. 5 neonates $(83.3 \%)$ were (SGA) while one neonate $(16.7 \%)$ was (LGA). The two patients with reversed EDF distributed equally as 50\% normal birth weight and 50\% SGA.

Table 4: Relationship between UA Doppler EDF velocimetry and birth weight.

\begin{tabular}{|c|c|c|c|c|c|}
\hline \multirow[b]{2}{*}{ EDF } & \multicolumn{3}{|c|}{ Birth of weight } & \multicolumn{2}{|c|}{ p-value } \\
\hline & $\begin{array}{l}\text { Normal } \\
N=73\end{array}$ & $\begin{array}{l}\text { SGA } \\
N=28\end{array}$ & $\begin{array}{l}\text { LGA } \\
\mathbf{N}=\mathbf{3}\end{array}$ & Total & \multirow{5}{*}{0.001} \\
\hline Normal & $77.3 \%$ & $22.6 \%$ & $0 \%$ & $100 \%$ & \\
\hline Reduced & $66.7 \%$ & $23.8 \%$ & $9.5 \%$ & $100 \%$ & \\
\hline Absent & $0 \%$ & $83.3 \%$ & $16.7 \%$ & $100 \%$ & \\
\hline Reversed & $50 \%$ & $50 \%$ & $0 \%$ & $100 \%$ & \\
\hline
\end{tabular}

Table 5: Relationship between UA Doppler EDF velocimetry and Apgar score.

\begin{tabular}{|lllll|}
\cline { 2 - 4 } EDF & $\begin{array}{l}\text { Apgar } \\
\text { Normal } \\
\text { N=62 }\end{array}$ & $\begin{array}{l}\text { Abnormal } \\
\mathbf{N}=\mathbf{4 0}\end{array}$ & $\begin{array}{l}\text { Total } \\
\text { p- } \\
\text { value }\end{array}$ \\
\cline { 1 - 4 } Normal & $72 \%$ & $28 \%$ & $100 \%$ & \\
\cline { 1 - 4 } Reduced & $38.1 \%$ & $61.9 \%$ & $100 \%$ & \multirow{2}{*}{0.00} \\
\cline { 1 - 4 } Absent & $0 \%$ & $100 \%$ & $100 \%$ & \\
\hline Reversed & $0 \%$ & $100 \%$ & $100 \%$ & \\
\hline
\end{tabular}

Regarding Apgar score at 5 minutes, there was significant relationship (p-value $=0.00)$ with UA Doppler EDF velocimetry (Table 5). Out of the 75 patients with normal EDF velocimetry; 54 neonates (72\%) had normal Apgar score while 21 neonates (28\%) had abnormal Apgar score. 8 neonates $(38.1 \%)$ with reduced EDF velocities had normal Apgar score while 13 neonates $(61.9 \%)$ had abnormal Apgar score. A total of 55 neonates (73.3\%) with normal EDF velocities were admitted to SCBU, while 20 neonates $(26.7 \%)$ were not. 8 neonates $(38.1 \%)$ with reduced EDF velocities were admitted to SCBU, while 13 neonates $(61.9 \%)$ were not as shown in Table 6. All neonates $(100 \%)$ with absent and reversed EDF velocities were admitted to SCBU. All neonates (100\%) with absent and reversed EDF velocities had abnormal Apgar score at 5 minutes.

Table 6: Relationship between UA Doppler EDF velocimetry and admission to SCBU.

\begin{tabular}{|lllll|}
\hline \multirow{2}{*}{ EDF } & \multicolumn{3}{l}{ Admission to SCBU } & \\
& $\begin{array}{l}\text { No } \\
\text { N=63 }\end{array}$ & $\begin{array}{l}\text { Yes } \\
\text { N=41 }\end{array}$ & Total & p-value \\
\cline { 1 - 4 } Normal & $73.3 \%$ & $26.7 \%$ & $100 \%$ & \\
\cline { 1 - 4 } Reduced & $38.1 \%$ & $61.9 \%$ & $100 \%$ & \multirow{2}{*}{0.00} \\
\cline { 1 - 4 } Absent & $0 \%$ & $100 \%$ & $100 \%$ & \\
\cline { 1 - 4 } Reversed & $0 \%$ & $100 \%$ & $100 \%$ & \\
\hline
\end{tabular}

In the current study, the UA Doppler EDF velocimetry was significantly changed with respiratory distress syndrome (RDS) and early neonatal death (END), pvalue $=0.00$ for both as shown in Table 7. A total of 67 neonates $(89.3 \%)$ with normal EDF velocities did not develop RDS; while 8 neonates $(10.7 \%)$ developed RDS. 15 neonates $(71.4 \%)$ with reduced EDF velocities did not develop RDS, while 6 neonates $(28.5 \%)$ developed RDS. All patients $(100 \%)$ with absent and reversed EDF velocities developed RDS. Regarding END, 3 neonates (4\%) with normal EDF velocities died early in the neonatal period, while 72 neonates $(96 \%)$ did not. 4 neonates $(19 \%)$ with reduced EDF velocities died early in the neonatal period, while 17 neonates $(81 \%)$ did not. 3 neonates $(50 \%)$ with absent EDF velocities died, while 3 neonates $(50 \%)$ did not. All neonates (100\%) with reversed EDF died in the first week of neonatal period.

Table 7: Relationship between UA Doppler EDF velocimetry with RDS and END.

\begin{tabular}{|lllll|}
\hline EDF & $\begin{array}{l}\text { RDS } \\
\text { No } \\
\mathbf{n = 8 2}\end{array}$ & $\begin{array}{l}\text { Yes } \\
\mathbf{n = 2 2}\end{array}$ & $\begin{array}{l}\text { No } \\
\mathbf{n = 9 2}\end{array}$ & $\begin{array}{l}\text { Yes } \\
\mathbf{n = 1 2}\end{array}$ \\
\hline Normal & $89.3 \%$ & $10.7 \%$ & $96 \%$ & $4 \%$ \\
\hline Reduced & $71.4 \%$ & $28.5 \%$ & $81 \%$ & $19 \%$ \\
\hline Absent & $0 \%$ & $100 \%$ & $50 \%$ & $50 \%$ \\
\hline Reversed & $0 \%$ & $100 \%$ & $0 \%$ & $100 \%$ \\
\hline p-value & 0.00 & & 0.00 & \\
\hline
\end{tabular}

\section{DISCUSSION}

The exact etiology of preeclampsia is unknown and probably complex and multi-factorial. Although the primary events leading to pre-eclampsia are still unclear, it is now widely believed that a cascade of events leads to this clinical syndrome. And an imposing number of mechanisms have been proposed to explain its causes. Instead of being simply "one disease", pre-eclampsia 
appears to be culmination of factors that likely involve a number of maternal, placental and fetal factors. 6 The clinical course of severe pre-eclampsia is marked by inevitable progression, which may be gradual or fulminant.

In the present study, the researchers observed that the EDV was abnormal in $27.9 \%$ of the participants; reduced in $20.2 \%$, absent in $5.8 \%$ and reversed in $1.9 \%$. Fetuses with abnormal EDV usually developed adverse perinatal outcomes. Some authors showed a relation between abnormal umbilical artery waveform and adverse neonatal outcomes.7 This relation used to decrease perinatal mortality and morbidity in high-risk obstetric situations. ${ }^{8}$ This current study intended to use the EDV alone since the other Doppler parameters were decline through the course of gestation due to progressive maturation of the placenta and increase in the number of tertiary stem villi. ${ }^{9}$

Regarding to the relationship between the onset time of pre-eclampsia and the UA Doppler velocimetry, the researchers observed that early onset of pre-eclampsia, 20-30 weeks of gestation, was associated with the highest incidence of abnormal UA velocimetry. Compared to late onset of pre-eclampsia, 36-41 weeks of gestation, 36 patients $(48 \%)$ with normal EDV fall in this category, 5 patients $(23 \%)$ with reduced EDV, one patient $(16.7 \%)$ with absent EDV, while no patient $(0 \%)$ with reversed EDV was found in late onset of pre-eclampsia group. These findings agreed with the results obtained from similar studies that reported that the early onset of preeclampsia is associated with increased rates of abnormal fetal Doppler velocimetry in all utero-placental and fetoplacental vessels including uterine artery, middle cerebral artery, ductus venosus and umbilical artery. ${ }^{10-12}$

Moreover, the researchers observed that the EDV is reduced or absent in the cases that ended by emergency cesarean delivery more than the group of vaginal delivery although there was not statistical significant $(\mathrm{P}$ - value $=0.275$ ). This can be explained by the fact that all participants of the study were candidates for termination of pregnancy due to the severity of the disease, and it is the cervical condition plus the fetal and maternal compromise that determine the mode of delivery, not UA Doppler findings alone.

The current study revealed that increased incidence of IUGR, as reflected with small for gestational age (SGA) neonates, was found in the abnormal UA Doppler groups with statistical significance $(\mathrm{P}$ value $=0.001)$. These findings agreed with Bhatt et al, who reported that $60 \%$ of patients with abnormal UA Doppler delivered IUGR babies. $^{10}$

Regarding the relationship between EDV and Apgar score in the preeclampsia patients, there was statistical significance $(\mathrm{P}$ value $=0.00)$. All patients with absent and reversed EDV had abnormal Apgar score of less than 7 at
5 minutes, while only 21 patients $(28 \%)$ of patients with normal EDV had abnormal Apgar score at 5 minutes. This is explained by the fact that placental insufficiency in severe pre-eclampsia is a major cause of neonatal hypoxia and RDS. These findings were consistent with Barunoday et al results who reported "low Apgar score was significantly associated with raised Doppler indices". ${ }^{13}$

Early neonatal death (END) is the dark end of the spectrum of adverse neonatal outcome. The more the abnormality of UA Doppler EDV velocimetry, the more the incidence of END. All neonates with reversed EDV $(100 \%)$ died within the 1 st week of neonatal period, half of the neonates $(50 \%)$ with absent EDV also died in the early neonatal period, compared to only $4 \%$ of neonates with normal EDV. The results showed high rates of neonatal mortality compared to the study done by Bhatt, et al, who found only $50 \%$ mortality rate in abnormal UA Doppler EDV. ${ }^{10}$

Finally, the researchers observed that umbilical artery Doppler EDV in preeclampsia was significantly associated with RDS (P-value $=0.00$ ). In our study, the EDF is reduced but not absent although several studies confirmed absence of EDV. ${ }^{14}$ Absent or reduced EDV of the UA reflect the degree of severity of RDS.

\section{ACKNOWLEDGEMENTS}

We are grateful to thank Dr. Susan Omer, for her guidance and help throughout the stages of this study and we also honored to thank Dr. Ahmed Alfatih and Najeeb Allah Abd.Rahim, who helped us in statistical analysis and preparing the study design.

\section{Funding: No funding sources Conflict of interest: None declared \\ Ethical approval: The study was approved by the Institutional Ethics Committee}

\section{REFERENCES}

1. Global Info Base. Geneva-WHO, 2014. Available at http://www.who-int/infobase/report. WHO aspx. Accessed 3 August 2016.

2. Lewis G. RCOG. The Six Report of the Confidential Enquiry into Maternal and Child Health. Why Mothers Die 2000-2002: London, 2004. Available at https://stratog.rcog.org.uk/files/rcogcorp/elearn/elearn_tra/Why-Mothers--Die-20002002_haemorrhage.pdf

3. Abass H. Suba University Hospital's perinatal morbidity and mortality meeting, 2001.

4. European Association of Perinatal Midicine. Regulation for the use of Doppler technology in perinatal medicine. In: consensus of Barcelona. Barcelona: Institute Barcelona; 1989:22-26. 
5. Alfirevic Z, Stampalija T, Gyte GM. Fetal and umbilical Doppler U/S in high risk pregnancies. Cochrane Data based syst Rev. 2010;1:CD007529

6. Dekker GA, Sibai BM. Etiology and pathogenesis of pre-eclampsia: current concepts. Am J Obstet Gynecol. 1998;179:1359-75.

7. American College of Obstetricians and Gynaecologists. Diagnosis and Management of Preeclampsia and Eclampsia: AOOG Practice Bulletin No. 33. Obstetrics and Gynecology. 2002;99:159-67.

8. Maulik D, Mundy D, Heitmann E. Evidence-based approach to umbilical artery Doppler fetal surveillance in high-risk pregnancies: an update. Clin Obstet Gynecol. 2010;53(4):869-78.

9. Avni KP Skandhan, Radswiki. Umbilical arterial Doppler assessment. Available at http://radiopaedia.org/articles/umbilical-arterialdoppler-assessment. Accessed 15 August 2016.

10. Batt CJ. Role of color Doppler in pregnancy induced hypertension, a study of 100 cases. Indian J Radial Imaging. 2003;13:417-20.

11. Todros T, Ronco G, Fianchino O, Rosso S, Gabrielli S. Accuracy of the umbilical arteries Doppler flow velocity waveforms in detecting adverse perinatal outcomes in a high-risk population. Acta Obstet Gynecol Scand. 1996;75(2):113-9.

12. Khalid M, Wahab S, Khalid VKS, Haroon S, Sabzposh NA. Doppler Indices in Prediction of Fetal Outcome in Hypertensive Pregnant Women. NJOG 2011;6(1):28-34.

13. Chakraborty B, Barman SC, Rudra BP, Sahana R, Mondal PC. Evaluation of Perinatal Outcome by Antenatal CTG and Umbilical Artery Doppler in Preeclamptic Mothers. Indian Journal of Clinical Practice. 2013;24(6):562-3.

14. Guan Y, Li S, Luo G, Wang C, Norwitz ER, Tu X, et al. The role of Doppler waveforms in the fetal main pulmonary artery in the prediction of neonatal respiratory distress syndrome. Journal of Clinical Ultrasound. 2015;43(6):375-83.

Cite this article as: Gameraddin MB, Gabo AA Umbilical artery velocity and adverse neonatal outcome in sudanese pregnant women with severe preeclampsia. Int J Reprod Contracept Obstet Gynecol 2016;5:4236-41. 